\title{
LOPH and D-MHC in the Treatment of FeLV Associated Acute Leukemia in a Cat
}

\author{
Rodrigo dos Santos Horta ${ }^{1}$, Ana Luisa Fajardo Ferreira', Mariana de Pádua Costa ${ }^{1}$, Ligia Soares Frossard², \\ Júlia Campero Nimrichter ${ }^{3}$, Mylena Duarte de Carli ${ }^{3} \&$ Paulo Ricardo de Oliveira Paes ${ }^{1}$
}

\begin{abstract}
Background: Bone marrow primary malignancies are denominated leukemias, classified as myeloid or lymphoid, according to the cell lineage, and acute or chronic, according to the cell's state of maturation. In cats, acute lymphoid leukemia is the most common form, especially in regions endemic for feline leukemia virus and / or feline immunodeficiency virus. A new treatment protocol for lymphomas, called LOPH, was described for animals with FeLV persistent viremia. This study aimed to report a case of a cat presenting with FeLV associated acute leukemia and treated with the LOPH protocol, and, in the rescue phase, a modification of the D-MAC protocol, denominated D-MHC.

Case: A 4-year-old mixed breed intact queen was attended due to lethargy and inappetence. The patient did not present any relevant abnormalities in the clinical exam and complementary exams were performed including complete blood count, biochemical profile, SNAP Feline Triple Test, chest radiographs and abdominal ultrasound. Imaging tests and biochemical values were unremarkable, but the patient presented a reagent result for FeLV and severe leukocytosis due to lymphocytosis. The morphological evaluation of the blood smear revealed the presence of blasts, in a concentration greater than $20 \%$ of the nucleated cells, which allowed the characterization of a leukemic state, probably lymphoid. First-line treatment was based on the LOPH protocol, including Lomustine, Vincristine, Prednisolone and Doxorubicin, in four-week cycles. Nevertheless, during the third cycle, 66 days after the institution of this protocol, the patient presented a febrile condition along with marked leukocytosis due to lymphocytosis, confirming leukemia recurrence. A rescue attempt was performed with a modification of the D-MAC protocol, originally consisting of the combination of dexamethasone, melphalan, actinomycin-D and cytarabine, but with replacement of actinomycin-D by doxorubicin, and therefore denominated D-MHC. After the three cycles there was a return of the febrile condition associated with severe pancytopenia and euthanasia was elected due to poor clinical condition, resulting in a survival time of 124 days. The hematological toxicity of the induction protocol included anemia and neutropenia, with mainly grade I events, but with the occurrence of a single grade IV event. The adverse effects of the rescue protocol were similar, but with a greater number of grade IV events.

Discussion: FeLV is considered the most lethal retrovirus of the domestic cat, with a major impact on health and life expectancy. Persistent FeLV antigenemia increases the risk of hematopoietic neoplasms in 62.1 times due to a direct insertional mutagenesis. In endemic regions, approximately $70 \%$ of cats with acute leukemia have persistent FeLV antigenemia, as the patient in this report. The diagnosis was made through association of retroviral status and the identification of more than $20 \%$ of blasts, possibly lymphoblasts, in the blood stream, sparing the need for a myelogram. Considering the poor prognosis for acute lymphoid leukemias and the patient's retroviral status, treatment was initiated with the LOPH protocol, including lomustine, as a potent agent to induce remission, and doxorubicin, which can result in longer remission interval. After 66 days free of the disease, the patient presented recurrence of the leukemic condition, starting the rescue protocol D-MHC. Remission was again obtained with duration similar to the first protocol, however, on the occasion of a new leukemia recurrence, euthanasia was elected. The treatment adopted for the patient in this report resulted in a longer survival time than reported in other studies. Despite the aggressiveness of the protocols, especially the D-MHC, it was possible to perform it using the monocyte and granulocyte stimulation factor to reverse neutropenia.
\end{abstract}

Keywords: oncology, chemotherapy, doxorubicin, lomustine, cytarabine, melphalan.

${ }^{1}$ Departamento de Clínica e Cirurgia Veterinárias, Escola de Veterinária, Universidade Federal de Minas Gerais (UFMG), Belo Horizonte, MG, Brazil. ${ }^{2}$ M.V. Autônoma, Belo Horizonte. ${ }^{3}$ Departamento de Medicina Veterinária, Universidade Vila Velha (UVV), Vila Velha, ES, Brazil. CORRESPONDENCE: R.S. Horta [rodrigohorta@ufmg.br / rodrigohvet@gmail.com]. Departamento de Clínica e Cirurgia Veterinárias, Escola de Veterinária, Universidade Federal de Minas Gerais (UFMG). Av. Pres. Antônio Carlos n. 6627. CEP31270-901 Belo Horizonte, MG, Brazil. 
R.S. Horta, A.L.F. Ferreira, M.P. Costa, et al. 2021. LOPH and D-MHC in the Treatment of FeLV Associated Acute Leukemia in a Cat. Acta Scientiae Veterinariae. 49(Suppl 1): 628.

\section{INTRODUCTION}

The term leukemia refers to malignant neoplastic proliferation originated in the body's blood-forming tissues (i.e., bone marrow), classified based on the cell lineage in myeloid or lymphoid, and considered acute, when they involve precursor cells, or chronic, when they involve mature cells [3,9]. Lymphoid leukemia, related to the malignant proliferation of lymphocytes and their precursors, is the most common form in humans, dogs and cats, although the real incidence remains unknown in Veterinary Medicine [3,6,9,31]. Chronic lymphoid leukemia is the most common in dogs, however, in cats, acute lymphoid leukemia, related to the proliferation of lymphoblasts and/or pro-lymphocytes, is the most common in endemic regions for the feline leukemia virus (FeLV) and / or feline immunodeficiency virus (FIV) [12].

A new treatment protocol has been described for multicentric or mediastinal lymphomas in cats with FeLV persistent antigenemia. This protocol, called $\mathrm{LOPH}$, is proposed for an induction phase and consists of the association of Lomustine, Vincristine (Oncovin $^{\circledR}$ ), Prednisolone and Doxorubicin (Hydroxydaunorubicin), showing good tolerance in cats, with complete response in $81 \%$ of cases and an average survival of 214 days, superior to studies with other protocols in populations with high FeLV infection rates [17].

In cases of disease recurrence, rescue therapies can be attempted, with emphasis on the D-MAC protocol, which originally consists of the combination of dexamethasone, melphalan, actinomycin-D and cytarabine [11].

This study aimed to report a case of a cat presenting with FeLV associated acute leukemia and treated with the LOPH protocol, and, in the rescue phase, a modification of the D-MAC protocol, denominated D-MHC.

\section{CASE}

A 4-year-old mixed breed intact queen was attended at a private practice due to lethargy and inappetence. The physical examination was unremarkable, however, complementary tests were carried out and included complete blood count, biochemical profile (urea, creatinine, alanine aminotransferase, aspartate aminotransferase, alkaline phosphatase, gamma-glutamyl transpeptidase, total protein and fractions, total bilirubin and fractions, calcium and SNAP Feline Triple Test ${ }^{1}$, chest radiographs and abdominal ultrasound. Imaging exams showed no changes. Biochemical results were unremarkable, but the patient presented a reagent result for FeLV and severe leukocytosis (125,500/ $\mu \mathrm{L}$; Ref: 3,000-14,800/ $\mu \mathrm{L}$ [18]) due to lymphocytosis (95\% of the relative count and

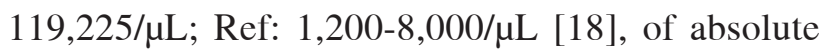
count), associated with normocytemia, with a packed cell volume (PCV) of $29.2 \%$ (Ref: $29-48 \%$ [18]) and platelet count within the reference values. In the morphological evaluation of the blood smear cell types, the presence of blast cells was found, in a concentration greater than $20 \%$ of the nucleated cells, which allowed the characterization of a leukemic state, probably lymphoid (Figure 1). As there were no changes in lymph nodes in physical and imaging exams, acute lymphoid leukemia was diagnosed, even in the absence of myelogram. Bone marrow aspirates were not performed due to the poor clinical condition of the patient and the need for anesthesia for the procedure.

With the diagnosis of acute lymphoid leukemia, chemotherapy treatment was initiated, and hematological toxicity was assessed based on the Veterinary

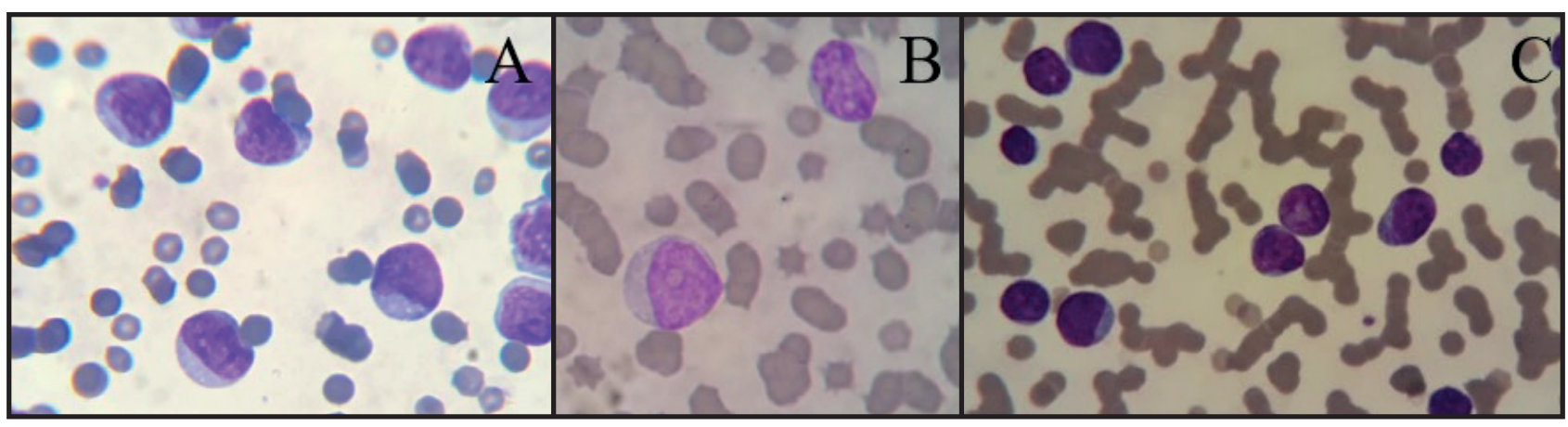

Figure 1. Peripheral blood smear showing blast cells, possibly lymphoid, of medium to large sizes (A, B, C), with frequently irregular or indented nuclei, with a finely granular chromatin pattern and prominent single or multiple nucleoli (B, C), and moderately basophilic cytoplasm with multivacuolizations (B, C) [Romanowski; 100x]. 
R.S. Horta, A.L.F. Ferreira, M.P. Costa, et al. 2021. LOPH and D-MHC in the Treatment of FeLV Associated Acute Leukemia in a Cat. Acta Scientiae Veterinariae. 49(Suppl 1): 628.

Table 1. Chemotherapy protocol for acute leukemia in a domestic cat, for induction it was used the LOPH protocol based on the combination of lomustine, vincristine $\left(\right.$ Oncovin $\left.^{\circledast}\right)$, prednisolone and doxorubicin (hydroxydaunorubicin) [17] and in a rescue attempt it was used the D-MHC protocol based on the combination of dexamethasone, melphalan, doxorubicin (hydroxydaunorubicin) and cytarabine [11].

\begin{tabular}{cc}
\hline Week & Drug \\
\hline & Induction Phase $($ Loph Protocol)* \\
\hline Week 1 & Lomustine $6 \mathrm{mg} /<3 \mathrm{~kg}, 9 \mathrm{mg} / 3-4,5 \mathrm{~kg}, 12 \mathrm{mg} / 4,5-7 \mathrm{~kg}, 15 \mathrm{mg} />\mathrm{kg} \mathrm{PO}$ \\
Week 2 & Vincristine $0,6 \mathrm{mg} / \mathrm{m}^{2} \mathrm{IP}$ \\
Week 3 & Doxorubicin $1 \mathrm{mg} / \mathrm{kg} \mathrm{IV} * *$ \\
Week 4 & Vincristine $0,6 \mathrm{mg} / \mathrm{m}^{2} \mathrm{IP}$ \\
\hline & Rescue Phase $($ D-Mhc Protocol)*** \\
\hline Week 1 & Doxorubicin $1 \mathrm{mg} / \mathrm{kg} \mathrm{IV}+$ Cytarabine $300 \mathrm{mg} / \mathrm{m}^{2} \mathrm{SC}+$ Dexamethasone $1 \mathrm{mg} / \mathrm{kg} \mathrm{SC}$ \\
Week 2 & Melphalan $20 \mathrm{mg} / \mathrm{m}^{2}$ PO + Dexamethasone $1 \mathrm{mg} / \mathrm{kg} \mathrm{SC}$ \\
\hline
\end{tabular}

*Repeat $5 \mathrm{x}$ (total 20 weeks); **Pre-medication with dexamethasone, at a dose of $0.5 \mathrm{mg} / \mathrm{kg}$, intravenously. ***Repeat indefinitely Prednisolone $10 \mathrm{mg}$ / cat / day, orally, continuous in the first cycle, followed by a gradual reduction from the second cycle ( $25 \%$ every 2 weeks).

Cooperative Oncology Group-Common Terminology Criteria for Adverse Events v.1.1 [33]

First-line treatment was based on the LOPH protocol [lomustine (magistral), vincristine (Onco$\left.\operatorname{vin}^{\circledR}\right)^{2}$, prednisolone $\left(\text { Prediderm }{ }^{\circledR}\right)^{3}$ and doxorubicin $\left.{ }^{4}\right]$ (Table 1). The blood count was repeated 2 days after the first chemotherapy session (lomustine), with a reduction of leukocytosis and lymphocytosis by more than $50 \%$, but with the installation of mild anemia (PCV of 24\%, Ref: $29-48 \%$; red blood cell count of $4.6 \times 106 / \mu \mathrm{L}$, Ref: $5.92-9.93 \times 10^{6} / \mu \mathrm{L}$; and hemoglobin concentration of 7.9 g/dL; Ref: 9.3-15.9 g/dL [18]), normochromic normocytic, with no anisocytosis and polychromasia and $3 \%$ of rubricytes and metarrubriticytes among total nucleated cells, characteristic of non-regenerative anemia probably due to bone marrow impairment or myelophitisis. A week after the first chemotherapy session, leukogram was normal, but there was worsening of the anemia, with reduction of PCV (18.5\%; Ref: $29-48 \%$ [18]), red blood cell count $\left(3.01 \times 10^{6} / \mu \mathrm{L}\right.$; Ref: $\left.5.92-9.93 \times 10^{6} / \mu \mathrm{L}[18]\right)$ and hemoglobin concentration (6.0 g/dL; Ref: 9.3-15.9 g/dL [18]), hypochromic macrocytic, with $1 \%$ of nucleated erythroid cells. In the third week of chemotherapy, a mild recovery from anemia (grade II) was observed, but there was marked leukopenia and neutropenia (grade IV). Oral antibiotic therapy with marbofloxacin (Marbopet $\left.{ }^{\circledR}\right)^{5}(3 \mathrm{mg} / \mathrm{kg})$ was initiated and a granulocyte and monocyte stimulating factor (i.e., filgrastim $\left(\text { Granulokine }{ }^{\circledR}\right)^{6}$ ), was administered subcutaneously, at a dose of $5 \mathrm{mcg} / \mathrm{kg}$, for 2 consecutive days. After $48 \mathrm{~h}$, an increase in neutrophil counts $(1840 / \mu \mathrm{L})$ was observed, allowing continuation of the chemotherapy protocol (doxorubicin ${ }^{4}$ ). In the following week, as the neutrophils remained close to the previous values, chemotherapy was continued, this time with the concomitant administration of a single dose of filgrastim. The second cycle of chemotherapy and the first session of the third cycle were performed, despite grade I-II neutropenia. Nevertheless, in the second session of the third cycle, the patient had a fever and chemotherapy was suspended until imaging tests were performed, to discard infection. There were no changes in imaging tests, but another complete blood count revealed, once more, marked leukocytosis

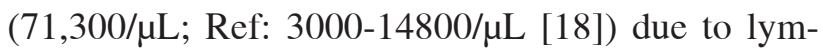
phocytosis $(81 \%$ of the relative count and $57753 / \mu \mathrm{L}$; Ref: $1200-8000 / \mu \mathrm{L}$, of absolute count [18]), confirming leukemia recurrence. Chemotherapy continued with the second session of this cycle (vincristine) ${ }^{2}$, and in the following 2 days, the administration of cytarabine $\left(\text { Citarax }^{\circledR}\right)^{7}$, subcutaneously, at a dose of $600 \mathrm{mg} / \mathrm{m}^{2}$, divided into 4 doses, every $12 \mathrm{~h}$.

With treatment, the febrile condition ceased and the patient's general condition improved, with resolution of lymphocytosis, but with the presence of severe neutropenia (820/ $\mu \mathrm{L}$; Ref: $2500-8500 / \mu \mathrm{L}$ [18]), requiring, again, institution of filgrastim, which allowed, in the following week, the institution of the rescue protocol D-MHC [dexamethasone $\left(\text { Dextar }^{\circledR}\right)^{8}$ melphalan $\left(\text { Alkeran }^{\circledR}\right)^{9}$, doxorubicin ${ }^{4}$, and cytarabine $\left.{ }^{7}\right]$ (Table 1), adapted from the D-MAC protocol, with the replacement of actinomycin-D by doxorubicin ${ }^{4}$ 
(hydroxydaunorubicin), of the same pharmacological group. The beginning of the rescue protocol was marked by mild anemia (PCV of 22.1\%; Ref: 29-48\% [18]), which was maintained throughout the treatment (3 cycles), along with episodes of neutropenia (grade III-IV). After the 3 cycles there was a return of the febrile condition associated with severe pancytopenia (PCV of $10.1 \%$, total leukocyte count of $100 / \mu \mathrm{L}$ and platelets count of $38 \times 103 / \mu \mathrm{L}$ ) and euthanasia was elected due poor clinical condition. Necropsy was not authorized.

The disease-free interval since the start of chemotherapy (induction phase) was 66 days and survival was 124 days. The LOPH protocol presented packed cell volume and neutrophils toxicity grade I 4 times and grades II and III once, in addition to grade I and III hemoglobin toxicity once. The D-MHC protocol presented packed cell volume toxicity grade I once, grade II 3 times and grade IV twice; hemoglobin toxicity grade I twice and grade II once; neutrophils toxicity grade III once and grade IV 4 times and platelets toxicity grade III once. During treatment, in the induction and rescue phases, filgrastim was used before 7 treatments ( 8 applications), in weeks 3, 4, 5, $8,9,11$ e 12 . Antibiotic therapy with marbofloxacin ${ }^{5}$ was instituted and maintained whenever the neutrophil count was below 1000/ $\mu \mathrm{L}$.

\section{DISCUSSION}

FeLV is considered the most lethal retrovirus of the domestic cat, with a major impact on the health and life expectancy [25]. Persistent FeLV antigenemia reduces the patient's life expectancy to 2.4 years, with only $20 \%$ of animals surviving after three years [19]. It is also associated with the occurrence of opportunistic infections and damage to target organs, and 15-23\% of cancer development [2,14,24-26]. FeLV infection increases the risk of hematopoietic neoplasms in 62.1 times, mainly lymphomas, but also leukemias [26]. The carcinogenic effect might be indirect, related to immunosuppression, with reduction of the antitumor response, however it is mainly related to a direct viral effect, characterized by insertional mutagenesis, and promotion of transcription of proto-oncogenes, with emphasis on the MYC $[2,14,24,26]$.

FeLV has a worldwide distribution, however, there has been a drastic reduction in its prevalence due to the institution of preventive measures, including vaccination, diagnosis and isolation of sick animals, in the United States, Canada and most parts of Europe
$[15,20]$. In spite of that, the virus remains widespread in underdeveloped countries, as in Brazil, with prevalence rates ranging from $8 \%$ to $63 \%$ in the states of São Paulo, Minas Gerais and Rio Grande do Sul [1,5,24,29]. In endemic regions, approximately $70 \%$ of cats with acute leukemia have persistent FeLV antigenemia [34], as in the patient in this report.

Historically, the diagnosis of acute leukemia is based on the identification of more than $30 \%$ of blasts in the peripheral blood (leukemic leukemia) or in the bone marrow, however, the cutoff point related to the identification of blasts in the peripheral blood has been reduced to $20 \%$ [6] in dogs, while the World Health Organization reduced this value to $20 \%$ in peripheral blood and bone marrow [3], although there is no consensus in Veterinary Medicine. In the study by Cotter [8], the diagnosis of acute lymphoid leukemia was established, in cats, in the presence of more than $50 \%$ of lymphoblasts in the bone marrow. However, other parameters must be considered, including confirmation of the lymphoid origin by flow cytometry or immunocytochemistry, as well as the occurrence of anemia, neutropenia and / or thrombocytopenia [6,31]. The diagnosis, in the present report, was made through the association of retroviral status, absence of identifiable lymphadenopathy and, based on the current literature [3,6,31], with the identification of more than $20 \%$ of blasts in the blood stream, sparing the need for a myelogram, whose sample collection must be made under general anesthesia, discouraged due to the patient's poor clinical condition.

Although flow cytometry is considered the gold standard for differentiation of lymphoid and myeloid precursors [9], this technique was not available at the time of care for this patient, as for immunocytochemistry. Histochemical stains could have been performed, considering that lymphoid precursors are usually negative for Sudan Black, myeloperoxidase and alkaline phosphatase, however, they have low specificity and staining with alkaline phosphatase can occur, albeit weaker, in neoplastic T lymphocytes [28]. Therefore, cell identification was based on morphology. As observed in this report, lymphoblasts are large cells with reduced nucleus:cytoplasm ratio basophilic cytoplasm and reduced granulation, regular nuclear membrane, indented and irregular nucleus, presence of prominent single or multiple nucleoli, being suggestive of acute lymphoid leukemia [16]. 
The lymphocytosis (119225/ $\mu \mathrm{L}$; Ref: 1500$7000 / \mu \mathrm{L}[18])$ observed in the patient of this report exceeds, with a wide margin of difference, that observed in reactive lymphocytosis, which rarely exceeds 30,000/ $\mathrm{L}$ [4]. The study by Tomiyasu et al. [31], included 6 cats with acute lymphoid leukemia, aged between 1 and 11 years (mean 5.5 years), with antibodies to FIV identified in 1 cat and 3 others showing FeLV antigenemia. Anemia was identified in 4/6 cats and thrombocytopenia in $5 / 6$, while leukopenia was observed in 2/6 cats and leukocytosis in 4/6, 3 of which had lymphocytosis greater than $40,000 / \mu \mathrm{L}$. The percentage of lymphoblasts in peripheral blood ranged from $5-97.5 \%$, and $5 / 6$ had lymphoblasts greater than $30 \%$. In the present report, despite the magnitude of lymphocytosis, anemia and thrombocytopenia were not observed in the first blood count. However, after the first chemotherapy session, there was a reduction in the lymphocyte count of almost $50 \%$, associated with a reduction in PCV (from $29.2 \%$ to $18.5 \%$ ), attributed to the reduction in the half-life of erythrocytes, due to the inflammatory process, possibly associated with myelophthisis and reduced bone marrow response. Recovery on subsequent exams was attributed to clinical remission of the disease and bone marrow regeneration [13]. A series of hematological abnormalities occurred as a result of the myelosuppressive effects of chemotherapy and disease recurrence.

Considering the poor prognosis for acute lymphoid leukemias [9] and the patient's retroviral status, treatment was initiated with the LOPH protocol, suggested by Horta et al. [17]. This protocol includes lomustine, as a potent agent to induce remission, considering its ineffectiveness in a possible rescue approach in cats with lymphoma, which resulted in a disease-free interval of only 39 days [10]. The LOPH protocol also includes doxorubicin in the induction phase. In underpowered studies, with a small number of cats with lymphoma, doxorubicin promoted longer remission intervals, when included in the induction $[17,32]$ or maintenance protocol [22].

In the present study, after 66 days free of the disease, the patient developed a fever that initially raised the suspicion of opportunistic infections, but with the sequential manifestation of lymphocytosis, the recurrence of the leukemic condition was evident, requiring the rapid establishment of a rescue protocol. For this, the D-MHC protocol was used, as a modification of the D-MAC protocol [11], considering the greater availability and easier acquisition of doxorubicin, which contributed to a faster initiation of the protocol. Cats have specific polymorphisms in the ABCG2 gene, resulting in the abnormal expression of their transcriptional product, the breast cancer resistance protein (BCRP), related to anthracyclines resistance, with consequent inefficient efflux of their substrates, such as doxorubicin [23], which favors, therefore, the use of this drug in the induction and rescue protocol [17]. The patient presented a new clinical remission, with duration similar to that observed with the first protocol, but there was a novel recurrence with severe pancytopenia, and euthanasia was elected.

The prognosis of acute lymphoid leukemia in dogs and cats is slightly better than the myloid counterpart, however, it remains poor and, although $20-40 \%$ of patients reach clinical remission with chemotherapy, this response is usually short, lasting 1-3 months [9]. The disease-free interval and survival in the present report were, however, higher than described in the literature, especially in cats [9,31]. In dogs and cats, the treatment of acute leukemia is usually carried out with the same protocols used for high-grade lymphomas, despite the worse prognosis and the intrinsic differences of each type of leukemia, whether lymphoid or myeloid and their subtypes $[9,31]$. The COP protocol, first described by Cotter [8], is the most widespread in cats, with its therapy being a combination of cyclophosphamide, vincristine (Onco$\left.\operatorname{vin}^{\circledR 2}\right)$ and prednisolone. The treatment of lymphoma results in complete remission in approximately $77 \%$ of cases, with a disease-free interval of 150 to 251 days and median survival of 266 days, although the best results are obtained in populations with reduced FeLV infection rate, which is related to a worse prognosis $[8,30,32]$. Regarding the viremic status of cats, in a study with the COP protocol in cats with lymphoma, with different clinical presentations, a median survival of only 37 days was observed for cats with persistent FeLV antigenemia [32], while those without detectable FeLV had a survival of 170 days. The CHOP protocol and its modifications are based on the addition of doxorubicin (hydroxydaunorubicin), however, lower rates of complete remission and survival are observed [7,21]. The Wisconsin-Madison protocol, originally described in 1996 as a CHOP-based protocol, was evaluated by Milner et al. [21], who observed a complete remission 
rate of only $47 \%$ and an average survival of 210 days. The inclusion of L-asparaginase, resulting in the L-CHOP protocol shows similar results with complete remission rate in $74 \%$ of cases and median survival of 232 days [27]. A study showed that retroviral status did not influence the survival of cats with acute lymphoid leukemia [31], however the study included only 6 cats and chemotherapy treatment was performed in only 5, with a median survival of 55 days. However, the chemotherapy protocol used was not uniform and included the use of prednisolone alone (33-days survival), L-CHOP (62-day disease-free interval) and the combination of L-asparaginase, cytarabine and nimustine (free interval $10^{6}$ days of illness).

The hematological toxicity of the LOPH protocol included anemia and neutropenia, which reached grade IV only once, maintaining mainly grade I, as reported by Horta et al. [17]. However, it was necessary to use filgrastim in certain stages of the protocol to allow continuity of treatment. Although its use in veterinary medicine is controversial, it is able to accelerate recovery and reduce the severity of neutropenia in dogs undergoing chemotherapy with cyclophosphamide [35]. In the D-MAC rescue protocol, the side effects found were similar, but in a greater number and more grade IV events.

Acute leukemia associated with FeLV is a neoplasm with a poor prognosis and complex treatment, with few evidences in the literature. The treatment of the patient in this report with the LOPH protocol resulted in a longer survival than that reported in other studies. In addition, the adaptation of the D-MAC protocol, called D-MHC, enabled the rapid institution of rescue, which also contributed to the greater survival of the patient. Both protocols had grade III and grade IV toxicity events, however, the LOPH protocol presented mainly grade I events, being well tolerated by the patient. The D-MHC protocol was shown to be a more aggressive protocol for the patient, with more grade III and IV toxicity events, but with satisfactory results, when associated with supportive treatments, such as the use of filgrastim and prophylactic antibiotic therapy.

\section{MANUFACTURERS}

${ }^{1}$ IDEXX Brasil Laboratórios Ltda. São Paulo, SP, Brazil.

${ }^{2}$ Antibióticos do Brasil Ltda. Cosmópolis, SP, Brazil.

${ }^{3}$ Ouro Fino Saúde Animal Ltda. Cravinhos, SP, Brazil.

${ }^{4}$ Eurofarma Laboratórios S.A. São Paulo, SP, Brazil.

${ }^{5}$ Ceva Saúde Animal Ltda. Paulínia, SP, Brazil.

${ }^{6}$ Amgen Biotecnologia do Brasil Ltda. Taboão da Serra, SP, Brazil.

${ }^{7}$ Blau Farmacêutica S.A. Cotia, SP, Brazil.

${ }^{8}$ União Química Farmacêutica Nacional S.A. São Paulo, SP, Brazil.

${ }^{9}$ Aspen Pharma Indústria Farmacêutica Ltda. Serra, ES, Brazil.

Acknowledgments. Anna Raquel Amaral for being such a lovely "mother" for her cat and consenting with this scientific publication.

Declaration of interest. The authors report no conflicts of interest. The authors alone are responsible for the content and writing of this paper.

\section{REFERENCES}

1 Almeida N.R., Danelli M.G.M., Silva L.H.P., Hagiwara M.K. \& Mazur C. 2012. Prevalence of feline leukemia virus infection in domestic cats in Rio de Janeiro. Journal of Feline Medicine and Surgery. 14(8): 583-586. DOI: 10.1177/1098612X12444693.

2 Almeida N.R., Soares L.D.\&Wardini A.B.W. 2016. Alterações clínicas e hematológicas em gatos domésticos naturalmente infectados pelo Vírus da Leucemia Felina (FeLV). Revista de Saúde, 7(1): 27-32. DOI: 10.21727/rs.v7i1.85.

3 Arber D.A., Orazi A., Hasserjian R., Thiele J., Borowitz M.J., Le Beau M.M., Bloomfield C.D., Cazzola M. \& Vardiman J.W. 2016. The 2016 revision to the World Health Organization classification of myeloid neoplasms and acute leukemia. Blood. 127(20): 2391-2405. DOI: 10.1182/blood-2016-03-643544.

4 Avery A.C. \& Avery P.R. 2007. Determining the Significance of Persistent Lymphocytosis. Veterinary Clinics: Small Animal Practice. 37(2): 267-282. DOI: 10.1016/j.cvsm.2006.11.001.

5 Barbosa F.C., Christianine M.P.T. \& Waldemarin K.C.A. 2001. Prevalência de Leucemia Felina em Gatos Doméstico de Uberlândia - MG. Arquivos de Ciências Veterinárias e Zoologia. 5(2): 207-211.

6 Bennett A.L., Williams L.E., Ferguson M.W., Hauck M.L., Suter S.E., Lanier C.B. \& Hess P.R. 2017. Canine acute leukaemia: 50 cases (1989-2014). Veterinary and Comparative Oncology. 15(3): 1101-1114. DOI: 10.1111/vco.12251.

7 Collette S.A., Allstadt S.D., Chon E.M., Vernau W., Smith A.N., Garret L.D., Choy K., Rebhun R.D., Rodriguez Jr C.O. \& Skorupski K.A. 2016. Treatment of feline intermediate to high-grade lymphoma with a modified university of Wisconsin-Madison protocol: 119 cases (2004-2012). Veterinary and Comparative Oncology. 14(1): 136-146. DOI: $10.1111 /$ vco. 12158 . 
R.S. Horta, A.L.F. Ferreira, M.P. Costa, et al. 2021. LOPH and D-MHC in the Treatment of FeLV Associated Acute Leukemia in a Cat. Acta Scientiae Veterinariae. 49(Suppl 1): 628.

8 Cotter S.M. 1983. Treatment of lymphoma and leukemia with cyclophosphamide, vincristine, and prednisone: II. Treatment of cats. The Journal of the American Animal Hospital Association. 19(2): 166-172.

9 Dobson J., Villiers E. \& Morris J. 2006. Diagnosis and management of leukaemia in dogs and cats. In Practice. 28(1): 22-31. DOI: 10.1136/inpract.28.1.22.

10 Dutelle A.L., Bulman-Fleming J.C., Lewis C.A. \& Rosenberg M.P. 2012. Evaluation of lomustine as a rescue agent for cats with resistant lymphoma. Journal of Feline Medicine Surgery. 14(10): 694-700. DOI: 10.1177/1098612X12448017.

11 Elliot J. \& Finotello R. 2018. A dexamethasone, melphalan, actinomycin-D and cytarabine chemotherapy protocol as a rescue treatment for feline lymphoma. Veterinary and comparative oncology. 16(1): 11-151. DOI: 10.1111/vco.12360.

12 Essex M.E. 1982. Feline leukemia: a naturally occurring cancer of infectious origin. Epidemiologic Reviews. 4: 189203. DOI: 10.1093/oxfordjournals.epirev.a036246

13 Fry M.M. \& McGavin M.D. 2013. Medula Óssea, Células Sanguíneas e Sistema Linfático. In: Zachary J. \& McGavin M. (Eds). Bases da Patologia em Veterinária. 6.ed. Rio de Janeiro: Elsevier, pp.1831-2026.

14 Hagiwara M.K., Reche Júnior A. \& Lucas S.R.R. 1997. Estudo clínico da infecção de felinos pelo vírus da leucemia felina em São Paulo. Revista Brasileira de Ciência Veterinária. 4(1): 35-38.

15 Hartmann K. 2012. Clinical Aspects of Feline Retroviruses: A Review. Viruses. 4(11): 2684-2710. DOI: 10.4322/ rbcv.2015.065.

16 Harvey J.W. 2001. Atlas of Veterinary Hematology: Blood and Bone Marrow of Domestic Animals. St. Louis: Saunders, $240 \mathrm{p}$.

17 Horta R.S., Souza L.M., Sena B.V., Almeida O.I., Jaretta T.A., Pimenta M.M. \& Reche Júnior A. 2020. LOPH: a novel chemotherapeutic protocol for feline high-grade multicentric or mediastinal lymphoma, developed in an area endemic for feline leukemia vírus. Journal of Feline Medicine and Surgery. 23(2): 86-97. DOI: 10.1177/1098612X20926893.

18 Klaassen J. 1999. Reference Values in Veterinary Medicine. Laboratory medicine. 30(3): 194-197.

19 Levy J.K., Scoot H.M., Lachtara J.L. \& Crawford P.C. 2006. Seroprevalence of feline leukemia virus and feline immunodeficiency virus infection among cats in North America and risk factors for seropositivity. Journal of the American Veterinary Medical Association. 228(3): 371-376.

20 Lutz H., Addie D., Belák S., Boucraut-Baralon C., Egberink H., Frymus T., Gruffydd-Jones T., Hartmann K., Hosie M., Lioret A., Marsilio F., Pennisi M.G., Radford A.D., Thiry E. Truyen U. \& Horzinek M.C. 2000. Feline Leukaemia ABCD guidelines on prevention and management. Journal of Feline Medicine and Surgery. 11(7): 565-574. DOI: 10.1016/j.fms.2009.05.005.

21 Milner R.J., Peyton J., Cooke K., Fox L.E., Gallangher A., Gordon P. \& HesterJ. 2005. Response rates and survival times for cats with lymhpoma treated with the University of Wisconsin - Madison chemotherapy protocol: 38 cases (1996-2003). Journal of the American Veterinary Medical Association. 227(7): 1118-1122. DOI: 10.2460/ javma.2005.227.1118.

22 Moore A.S., Cotter S.M., Frimberger A.E., Wood C.A., Rand W.M. \& L'Heureux D. 1996. A comparison of Doxorubicin and COP for Maintenance of Remission in Cats with Lymphoma. Journal of Veterinary Internal Medicine. 10(6): 372-375.

23 Ramirez C.J., Minch J.D., Gay J.M., Lahmers S.M., Guerra D.J., Haldorson G.J., Schneider T. \& Mealey K.L. 2011. Molecular genetic basis for fluoroquinolone-induced retinal degeneration in cats. Pharmacogennet Genomics. 21(2): 66-75. DOI: 10.1097/FPC.0b013e3283425f44.

24 Reche Júnior A., Hagiwara M.K. \& Lucas S.R.R. 1997. Clinical study of acquired immunodeficiency syndrome in domestic cats in São Paulo. Brazilian Journal of Veterinary Research and Animal Science. 24(3): 152-155.

25 Reinacher M. 1989. Diseases Associated with Spontaneous Feline Leukemia Virus (FeLV) Infection in Cats. Veterinary Immunology and Immunopathology. 21(1): 85-95. DOI: 10.1016/0165-2427(89)90132-3.

26 Shelton G.H., Grant C.K., Cotter S.M., Gardner M.B., Hardy W.D. \& DiGiacomo R.F. 1990. Feline Immunodeficiency Virus and Feline Leukemia Virus Infections and their Relationships to Lymphoid Malignancies in Cats: A Retrospective Study (1968-1988). Journal of Acquired Immune Deficiency Syndromes. 3(6): 623-630.

27 Simon D., Eberle N., Laacke-Singer L. \& Nolte I. 2008. Combination chemotherapy in feline lymphoma: treatment outcome, tolerability, and duration in 23 cats. Journal of Veterinary Internal Medicine. 22(2): 394-400. DOI: 10.1111/j.1939-1676.2008.0057.x. 
28 Stokol T., Schaefer D.M., Shuman M., Belcher N. \& Dong L. 2015. Alkaline phosphatase is a useful cytochemical marker for the diagnosis of acute myelomonocytic and monocytic leukemia in the dog. Veterinary Clinical Pathology. 44(1): 79-93. doi:10.1111/vcp.12227

29 Teixeira B.M., Rajão D.S., Haddad J.P., Leite R.C. \& Reis J.K.P. 2007. Ocorrência do vírus da imunodeficiência felina e do vírus da leucemia felina em gatos domésticos mantidos em abrigos no município de Belo Horizonte. Arquivo Brasileiro de Medicina Veterinária e Zootecnia. 59(4): 939-942. DOI: 10.1590/S0102-09352007000400019.

30 Teske E., Straten G., Noort R. \& Rutterman G.R. 2002. Chemotherapy with cyclophophamide, vincristine, and prednisolone (COP) in cats with malignant lymphoma: new results with an old protocol. Journal of Veterinary Internal Medicine. 16(2): 179-186. DOI: 10.1892/0891-6640(2002)016<0179:cwcvap>2.3.co;2.

31 Tomiyasu H., Doi A., Chambers J.K., Goto-Koshino Y., Ohmi A., Ohno K. \& Tsujimoto H. 2018. Clinical and clinicopathological characteristics of acute lymphoblastic leukaemia in six cats. Journal of Small Animal Practice. 59(12): 742-746. DOI: 10.1111/jsap.12917.

32 Vail D.M., Moore A.S., Ogilvie G.K. \& Volk L.M. 1998. Feline Lymphoma (145 Cases): Proliferation Indices, Cluster of Differentiation 3 Immunoreactivity, and Their Association with Prognosis in 90 Cats. Journal of Veterinary Internal Medicine. 12(5): 349-354. DOI: 10.1111/j.1939-1676.1998.tb02134.x.

33 Veterinary cooperative oncology group. 2016. Common terminology criteria for adverse events (VCOG-CTCAE) following chemotherapy or biological antineoplastic therapy in dogs and cats v1.1. Veterinary Comparative Oncology. 14(4): 417-446. DOI: 10.1111/vco.283.

34 Weijer K. \& Daams J.H. 1976. The presence of leukaemia (lymphosarcoma) and feline leukaemia virus (FeLV) in cats in The Netherlands. Journal Small Animal Practice. 17(10): 649-659. DOI: 10.1111/j.1748-5827.1976.tb06925.x.

35 Yamamoto A., Fujino M., Tsuchiya T. \& Iwata A. 2011. Recombinant canine granulocyte colony-stimulating factor accelerates recovery from cyclophosphamide-induced neutropenia in dogs. Veterinary immunology and immunopathology. 142(3-4): 271-275. DOI: 10.1016/j.vetimm.2011.05.021. 